\title{
De la Guerra Fría a la Sociedad Global. Las Relaciones Científicas Chileno-Alemanas 1949-2015: Contexto Internacional y Dinámicas del Intercambio
}

\author{
Enrique Fernández Darraz \\ Doutor em Sociologia pela Universidade Livre de Berlim. Pontificia Universidad Católica de Chile, \\ Santiago, Chile. \\ E-mail: efernandezdarraz@gmail.com, https://orcid.org/0000-0003-2356-1663
}

\section{INTRODUCCIÓN}

$\mathrm{C}^{1}$ presente artículo analiza las dinámicas que han seguido las Guerra Mundial hasta hoy, situándolas en el marco de la discusión sobre la circulación de conocimiento y las relaciones científicas internacionales.

Entre ambos países ha existido un largo y estrecho vínculo, sólo comparable al que Alemania ha mantenido con México, Brasil y Argentina, no obstante la evidente superioridad en tamaño y relevancia internacional de dichos países.

Las relaciones chileno-alemanas han sido estudiadas extensamente, pero a pesar de ello perviven en la literatura tres vacíos relevantes. El primero es la escasa investigación acerca de sus relaciones científicas. El segundo es la ausencia de un tratamiento políticamente integrado, que aborde tanto las relaciones de la República Federal (RFA), como las de la República Democrática Alemana (RDA) con Chile. Por último, tampoco existen investigaciones que contextualicen las relaciones científicas y las vinculen a procesos históricos y políticos mayores. Debido a estas razones no se cuenta con una interpretación más general, que permita explorar las significaciones de dicho intercambio científico.

DADOS, Rio de Janeiro, vol.62(4):e20160093, 2019. 
Este artículo intenta llenar estos vacíos, ofreciendo un análisis de las relaciones científicas en una perspectiva de largo plazo, integrando a todos los actores involucrados y situándolas en un marco de política internacional más amplio. El texto propone, además, una periodificación que permite comprender las dinámicas que éstas han seguido e identificar algunos elementos contextuales que han incidido en ellas.

Para desarrollar este análisis se realizó una extensa revisión de material de archivo referido a los acuerdos científicos intergubernamentales e interinstitucionales, a partir de las Memorias del Ministerio de Relaciones Exteriores chileno. También se revisaron documentos del Archivo Federal alemán sobre las relaciones internacionales del Partido Socialista Unificado (PSUA) de la extinta RDA, dado que no existen estudios sistemáticos de la política científica en dicho país. También se consultó prensa de ambos países y, para las décadas recientes, se contó con estadísticas de chilenos que han estudiado en Alemania.

Dado que el propósito del texto es caracterizar las relaciones científicas, la utilización del material de archivo, prensa y estadísticas no es exhaustiva, sino que se usó para ilustrar las dinámicas que estas han seguido en distintos momentos del último medio siglo.

Subyace a este artículo la hipótesis que, si bien existen asimetrías estructurales de larga data en la producción e intercambio científico, condiciones históricas y políticas específicas, así como intereses nacionales estratégicos, pueden condicionar la intensidad, forma y contenido de las relaciones científicas internacionales.

\section{CENTRO Y PERIFERIA: LAS ASIMETRÍAS DEL INTERCAMBIO CIENTÍFICO}

La discusión acerca de las asimetrías en la producción e intercambio de conocimiento tiene una importante tradición en la sociología del conocimiento. Su origen se vincula a la Teoría de la Dependencia y a algunos trabajos pioneros que, en la segunda mitad de la década de 1970, investigaron las relaciones de subordinación existentes en el mundo académico (Beigel, 2013b:18). Esta producción ha ido dando lugar a conceptos como el de geopolítica del conocimiento y a las tesis de la dependencia académica y del imperialismo académico o cultural, que buscan interpretar las dinámicas seguidas por los intercambios científicos y la circulación de saberes a nivel global (Alatas, 2003). 
Las dimensiones en que se ha abordado este tema han sido muchas. Más cercano al carácter principalmente económico de la Teoría de la Dependencia se encuentra, por ejemplo, el trabajo de Herrera (1991), que enfatiza la relación entre dependencia y desarrollo científico y económico nacional. En el otro polo se ubican investigaciones que buscan desentrañar las implicancias epistemológicas de los procesos de colonización, particularmente en las ciencias sociales periféricas $\mathrm{y}$, en especial, en la Sociología (Alatas, 2000:82).

Las tesis del eurocentrismo surgidas de estas últimas reflexiones, han intentado explicar no sólo cómo las ciencias sociales han transmitido sistemas de categorías que operan legitimando el saber de los países centrales, relegando a un segundo plano la producción local, sino también cómo han formado parte del aparato de dominación (Patel, 2015:45; Martínez, 2011:9-10; Walsh, 2001:66). Su función ha sido la de generar discursos que contribuyan a imponer interpretaciones y legitimar la institucionalidad colonial, incluso una vez constituidos los estados nacionales (Martínez, 2011:11).

Estos trabajos han abordado, además de los aspectos epistemológicos del eurocentrismo, los mecanismos y dispositivos específicos que han ido manteniendo y consolidando las situaciones de dependencia y dominación académica (Alatas, 2000:84). Por una parte, identifican como elementos decisivos de los países centrales su capacidad de producir grandes cantidades de artículos científicos, de acceder globalmente a las ideas e información que nutren dichos artículos, de influir en las ciencias sociales locales a través del consumo de sus artículos en la periferia y de gozar de un gran prestigio local y global, incluso más allá de su performance (Schott, 1993:204; Keim, 2008; Rodríguez-Medina, 2014:19).

Esta capacidad superior de producción se ve posibilitada por su volumen de capital intelectual, humano y de recursos financieros, que se expresan no sólo en equipamiento científico de vanguardia, sino también en archivos, bibliotecas, editoriales y revistas, entre otros (Patel, 2015:45-46). Contribuye a esta situación el hecho que agencias de gobierno, instituciones académicas y científicos locales se guían por criterios generados en los países centrales y orientan su carrera en función de un prestigio global que implica tributar temática o idiomáticamente a la producción de dichos países (RodríguezMedina, 2014:13; Beigel, 2013a:118-119). 
Estudios recientes han señalado al sistema de publicaciones como unos de los ejes de la actual forma de dominación y dependencia académica (Beigel, 2013a:112). La cienciometría se ha constituido en uno de los principales mecanismos de medición del prestigio científico global e incluso se ha extendido a las políticas públicas de educación superior y ciencia de muchos países, transformándose en un criterio relevante en los procesos de acreditación o en la asignación de recursos fiscales (Beigel, 2016:9). Esta métrica, sin embargo, consolida las asimetrías existentes, en la medida que ha sido construida sobre índices de publicaciones como ISI, Scopus $\mathrm{u}$ otras, que reflejan principalmente las publicaciones producidas en los países centrales (Beigel, 2013a:122; Beigel, 2013b:30).

Desde sus orígenes hasta hoy, las tesis del imperialismo y dependencia académica han ido complejizando su aparato conceptual, pasando de una visión binaria (centro-periferia) enraizada en el concepto de sistema mundo, a otras más complejas que identifican - para el caso de las ciencias sociales - a potencias semiperiféricas (Alatas, 2003:606), intentan comprender de modo menos mecánico la diversidad de las dinámicas de producción y circulación de conocimiento y se articulan en torno al concepto de Sistema Científico Internacional o Sistema Académico Mundial (Beigel, 2013b:19; Costa, 2014:235; Martínez, 2011:9-10).

A pesar que la utilización de los índices cientométricos continúa provocando una intensa acumulación de capital académico en los países centrales, el proceso de globalización ha provocado el surgimiento de nuevos actores científicos internacionales. De ese modo, por ejemplo, China se ha situado como el segundo productor de artículos científicos, luego de Estados Unidos; la movilidad estudiantil ha aumentado y se ha dirigido ya no sólo países habituales; y se han intensificado las colaboraciones que trascienden el eje Norte-Sur, generando importantes flujos en el sentido Sur-Sur y abriendo opciones para avanzar, incluso, en el nivel epistemológico hacia una superación del colonialismo existente (Beigel, 2013b:16; Dussel y Fornazzari, 2002:236; Patel, 2015:36). En las publicaciones, sin embargo, ello se aprecia principalmente en las ciencias naturales y menos en las sociales y humanidades (Beigel, 2013a:116).

Esta investigación busca contribuir a este debate desde el análisis de un caso específico: las relaciones científicas chileno-alemanas 
de los últimos 60 años. Particular relevancia se le da al vínculo entre las dinámicas de dichas relaciones y los contextos políticos internacionales específicos. De igual modo, se exploran algunas de las significaciones que el proceso de globalización y las nuevas ideas sobre la internacionalización de la ciencia han tenido en el intercambio académico de dos socios tradicionales, como han sido Alemania y Chile.

\section{LA INVESTIGACIÓN SOBRE LAS RELACIONES CIENTÍFICAS ENTRE ALEMANIA Y CHILE}

Las relaciones entre Chile y Alemania tienen una tradición que se remonta a la época de la conquista, pero adquieren un carácter sistemático con la llegada de un importante contingente de colonos alemanes a mediados del siglo XIX. En más de 150 años, dichas relaciones han sido profusamente estudiadas (Birle y Fernández, 2003). No obstante, no existen investigaciones que aborden de manera específica las relaciones científicas para un período extenso de tiempo.

Uno de los ámbitos tratados ha sido la contratación de profesores alemanes a fines del siglo XIX y su importancia en el desarrollo de la educación (Alarcón, 2005, 2010, 2014; Gutiérrez y Gutiérrez, 2004; Mellafe et al., 2007; Sanhueza, 2010, 2011, 2012). Sin embargo, estos estudios no tienen continuidad y se acotan al proceso mencionado.

Las Ciencias Sociales y Humanidades son las únicas que cuentan con una investigación sobre las relaciones científicas, en la publicación de Birle y Fernández (2003), pero esta no trasciende el carácter de una compilación y analiza sólo cuatro disciplinas: Ciencia Política, Historia, Sociología y Economía. La investigación de Holtz (2011), en tanto, se focaliza en los estudios de doctorado de chilenos, tanto en la RFA como en la RDA.

Para las ciencias naturales y tecnologías no se cuenta con investigaciones similares ${ }^{1}$. La Fundación Alexander von Humboldt (2009) publicó un Documento de Trabajo titulado "Cooperación científica chileno-alemana: Tradición y Futuro", que reúne artículos relativos a la cooperación científica entre ambos países. Sin embargo, estos no corresponden a indagaciones sistemáticas, sino a análisis de políticas y experiencias de intercambio. 
De igual modo, instituciones como el Deutscher Akademischer Austauschdienst (DAAD), publican informes anuales de su trabajo de colaboración científica desde la década de 1950, además de realizar estudios puntuales sobre algunos países de América Latina (DAAD, 2007).

Respecto de la relación de la RDA con Chile, la situación es similar. El trabajo de Drechsler (1978) aborda las relaciones políticas, culturales y científicas de la RDA con América Latina y Chile. Sin embargo, además de haber perdido actualidad, entrega sólo una visión panorámica de las mismas. Álvarez (2006) trata a los académicos chilenos en la Universidad de Leipzig de manera tangencial y en la reciente investigación de Emmerling (2013) el vínculo científico entre ambos países tiene menciones ocasionales.

Como se aprecia, la literatura sobre las relaciones científicas entre Chile y Alemania es exigua y en ella la pregunta por las dinámicas que estas han seguido no ha sido atendida.

\section{CHILE Y LAS DOS ALEMANIA. DEL FIN DE LA SEGUNDA GUERRA MUNDIAL AL GOLPE MILITAR}

El primer período posible de identificar en las relaciones científicas chileno-alemanas transcurre entre el fin de la Segunda Guerra, en 1945, y el golpe militar en Chile, en 1973.

Desde la perspectiva alemana, este período estuvo condicionado por dos aspectos: la división de Alemania en dos países y la competencia de ambos por lograr reconocimiento internacional. Desde el punto de vista de Chile, el elemento más relevante fue el avance de las ideas de izquierda, que provocó un acercamiento hacia el llamado bloque oriental.

En este periodo es posible distinguir dos momentos. El primero se extiende desde el fin de la Segunda Guerra hasta la Revolución Cubana, en 1959. El segundo fue inaugurado por ésta y culmina con dos hechos: la firma del tratado de reconocimiento mutuo de ambas Alemania, en 1972, y el golpe militar en Chile, en 1973. El primer hito puso fin a la competencia internacional de las dos Alemania, el segundo provocó un cambio profundo en la relación de estas con Chile. 


\section{Desde el fin de la Segunda Guerra a la Revolución Cubana}

El fin de la Segunda Guerra dejó a Alemania dividida en cuatro zonas de ocupación administradas por las potencias vencedoras: Estados Unidos, Francia, Inglaterra y la Unión Soviética.

Al llegar a 1949, las diferencias entre los aliados y la URSS habían escalado a tal punto que culminaron transformando a Alemania en dos países distintos, alineados correspondientemente con los dos bloques de la Guerra Fría. La República Federal Alemana en territorio occidental y la República Democrática Alemana, al oriente.

Ambos países desarrollaron una activa política internacional, condicionada por la conocida Doctrina Hallstein. En lo central, esta consistía en que Alemania Federal se arrogaba la continuidad política del Imperio y la representación del pueblo alemán. A la RDA la consideraba una zona de ocupación de la Unión Soviética, sin soberanía para representar a sus habitantes. Bajo esta premisa, hasta comienzos de la década de 1970, la RFA trató de impedir todo intento de la RDA por establecer relaciones diplomáticas más allá de los países socialistas.

La forma en que la RFA y la RDA enfrentaron las relaciones con América Latina y, en particular con Chile, fue distinta. Ambas tuvieron en común, sin embargo, que no asignaron importancia estratégica a la región.

Una de las primeras tareas que la República Federal acometió después de la elección de Konrad Adenauer como canciller (1949) fue reinsertarse internacionalmente. En esto, la promoción de relaciones científicas jugó un rol fundamental (Jöns, 2002, 2009; Schütte, 2010). En ese contexto, y como parte de la reconstrucción del Estado, la República Federal restableció la institucionalidad existente antes de la Guerra, destinada a promover el desarrollo científico y las relaciones académicas internacionales. Se fundó el Instituto Max Planck (1948), la Sociedad Fraunhofer (1949), el Servicio Alemán de Intercambio Académico (1950), la Sociedad Alemana de Ciencia (1951), el Instituto Goethe (1952) y la Fundación Alexander von Humboldt (1953) (Jöns, 2002, 2009). 
Alemania Democrática eligió en 1949 a Wilhelm Pieck como Primer Ministro y su gobierno se abocó a consolidar la nueva institucionalidad y a realizar reformas económicas y sociales acordes con el ideario socialista (Mählert, 2010). Internacionalmente la RDA estableció relaciones con países de la Unión Soviética e hizo un creciente esfuerzo por acercarse a los entonces conocidos como países no alineados, poniendo énfasis en las relaciones comerciales, como un primer paso para establecerlas a nivel diplomático (Wentker, 2007). La RDA fomentó en los primeros años el desarrollo de la ciencia y tecnología como un factor de producción económica. Hacia mediados de la década del 1950 fundó la Comisión Científica, para propiciar el desarrollo de la investigación. Sin embargo, el Partido Socialista Unificado Alemán (PSUA) conservó una fuerte influencia en la política científica (Lengwiler, 2010:18-19).

Chile mantuvo en este período una política internacional ajustada a su tradición y motivada por restablecer prontamente relaciones con Alemania. Para todos los efectos, al menos hasta mediados de la década de 1960, se sobreentendía que ello constituía una continuidad histórica y que el interlocutor era la República Federal. Especial relevancia tuvo en esta tarea el gobierno de Ibáñez (1952-1958), quien era un reconocido admirador de la RFA (Villarroel, 2007). A ello se debe agregar el extendido sentido anticomunista de la mayoría de la elite chilena que veía a la RDA con distancia (Dufner, 2012:64).

El gobierno siguiente, de Jorge Alessandri, conservó un vínculo estrecho con Alemania y mantuvo el alineamiento con los países occidentales (Quezada, 1983).

Si bien Chile y RFA reanudaron sus relaciones diplomáticas en 1951, en mayo de 1949 firmaron un primer acuerdo comercial (Dufner, 2012). Durante este período, ambos países concretaron 20 acuerdos, la gran mayoría comerciales y financieros, y sólo dos de cooperación científico-técnica (Arcos, 1989:64). El primero referido a "Ayuda Técnica" (MRREE, 1958:1029) y el segundo a "Cooperación Económica y Técnica" (MRREE, 1969:784). Ambos fijan las condiciones para el trabajo de especialistas alemanes en Chile.

Desde el punto de vista de las relaciones científicas, el convenio más importante se firmó en 1956, bajo el título de "Convenio Cultural" (MRREE, 1956:160-163). No obstante el nombre, el acuerdo está dedicado a 
fomentar el intercambio de catedráticos, profesores auxiliares, lectores, ayudantes, estudiantes y profesionales, facilitándoles sus viajes y otorgándole anualmente becas para que puedan complementar o perfeccionar sus estudios en régimen de intercambio (MRRE, 1956:160).

El tratado establecía condiciones para el intercambio académico, científico y cultural. Para concretarlo creaba dos comisiones mixtas, radicadas en Santiago y en Bonn, y tenía una duración de cinco años.

Con anterioridad a este "Convenio Cultural", la Memoria del año 1955 del Ministerio de Relaciones Exteriores registra la firma de dos convenios a nivel institucional, entre la Universidad de Chile y la Fundación Alemana Ibero-América (Deutsche Iberoamerika Stiftung), con motivo de la visita del Rector Juan Gómez Millas a la RFA. Sin embargo, salvo una breve referencia al "intercambio de profesores e investigadores", no especifica en qué consistía (MRREE, 1955:237).

Un año más tarde, el Presidente de la Conferencia de Rectores de las Universidades de Alemania Federal visitó Chile y, en particular, la Universidad Federico Santa María. En 1958 se firmó el Acuerdo de Científicos Visitantes destinado a fortalecer la cooperación entre las universidades chilenas y las de la RFA (Villarroel, 2007:81).

En esta etapa, el intercambio científico entre Chile y la RDA fue prácticamente inexistente. Dado, además, que ambos países establecieron relaciones diplomáticas en 1971, no se firmaron acuerdos bilaterales.

El principal vínculo fue comercial y se inició en 1952, cuando la República Democrática definió a Chile como clave para la obtención de materias primas y para su comercio internacional. Esto llevó a que en 1955 estableciera en Santiago una representación comercial, cerrada dos años más tarde (Dufner, 2007:32-33)².

\section{De la Revolución Cubana al Golpe de Estado de 1973}

El interés por Latinoamérica aumentó a medida que se consolidaron las posiciones geopolíticas de la Guerra Fría y, en especial, luego de la revolución cubana, que transformó al continente en centro de atención ya no sólo para Estados Unidos, sino también para la URSS y Europa (Krämer, 2011). 
En la región, esto activó la disputa por el reconocimiento internacional que ambas Alemania libraban en los países no alineados de África y el mundo árabe (Blumenau, 2011; Kilian, 2001).

La RDA comenzó una aproximación hacia América Latina a mediados de los 50, firmando acuerdos comerciales y financieros con Uruguay y Argentina (1954), Colombia y Chile (1955), y Brasil (1958). Luego abrió representaciones comerciales en esos y otros países: Uruguay (1954), Colombia, Argentina y Chile (1955) y Brasil (1959) (Krämer, 2011:184-185; Werz, 2011:447-448).

Su relación con América Latina se intensificó en los años 1960, luego que definiera como "estratégicos" a Brasil y a aquellos países que se abstuvieron de votar a favor de la expulsión de Cuba de la OEA, en 1962: Argentina, Bolivia, Chile, Ecuador y México (Krämer, 2011:185-186) ${ }^{3}$.

La presencia de Alemania Federal en el continente era privilegiada por los países, dada su importancia financiera y comercial. Por lo mismo, no tuvo dificultades para establecer relaciones diplomáticas con la mayoría de ellos (Hofmeister, 1998:60-61). Esto le facilitó la aplicación de la doctrina Hallstein. Un ejemplo ocurrió en 1961, cuando Brasil intentó un acercamiento a la RDA. Alemania Federal le hizo ver que ello tendría implicancias en sus relaciones y en la ayuda al desarrollo. Como consecuencia, Brasil no continúo con su acercamiento a Alemania Oriental (Rebmann, 2010).

Chile, por su parte, había comenzado un viraje hacia los países socialistas, intentando reducir su dependencia de Estados Unidos. En 1964 estableció relaciones diplomáticas con la Unión Soviética y, en 1965, con la República Popular de Polonia, la República Socialista de Checoslovaquia, la República Popular de Hungría, la República Popular de Bulgaria y la República Socialista de Rumania (Pedemonte, 2010:70-71).

A pesar de ello, es posible afirmar que Chile mantuvo hasta fines del gobierno de Eduardo Frei un alineamiento con los países occidentales, expresado en un mayor volumen de tratados, personal diplomático e intercambio comercial (Quezada, 1983:55).

El punto culminante de este giro tuvo lugar en el gobierno de Salvador Allende, que extendió las relaciones diplomáticas a otros países 
socialistas: China, Corea, Vietnam del Norte y la RDA (Kaufman, 1976:269). Esta situación, unida a la nacionalización de empresas extranjeras, tensionó los vínculos con los países occidentales y tuvo importantes consecuencias, incluida la pérdida de apoyo crediticio de Estados Unidos y, en el caso de la RFA, la suspensión de su ayuda al desarrollo, en 1971. No obstante, un año más tarde, en el marco de las negociaciones con el Club de París, la RFA retomó su programa de ayuda (Kaufman, 1976:265; Quezada, 1983).

También en Chile se intensificó la competencia entre ambas Alemania, conservando una de sus principales características: mientras la RFA gozaba de los beneficios de las relaciones diplomáticas, la RDA continuó trabajando a nivel no gubernamental, hasta 1971.

Durante este período Chile firmó un total de 37 acuerdos con Alemania Federal y 11 con Alemania Oriental (todos entre 1971 y 1973). Un cambio importante respecto al período anterior es que casi el $40 \%$ (14) de los firmados con la RFA y un 55\% (6) de los firmados con la RDA corresponden al área científico técnica (Arcos, 1989:64; MRREE, 1960-1973).

En estos acuerdos, el ámbito científico se encontraba unido a la actividad cultural y técnica. Todo ello, además, se subsumía con frecuencia dentro del concepto de "ayuda al desarrollo".

Un ejemplo que muestra cómo se entrelazaban fueron los convenios con la RFA para la creación de la Escuela Industrial Superior ChilenoAlemana de Ñuñoa. En ellos, la RFA se comprometía a enviar "El Director técnico y seis profesores o maestros instructores, por un período de tres años" (MRREE, 1964:133). Una situación similar se aprecia en el Acuerdo sobre Cooperación Económica de 1968. En él, además del envío de expertos, la RFA comprometía otorgar “...a chilenos, becas para centros de enseñanza técnica en diversos niveles" (MRREE, 1968:164).

El acuerdo científico más importante del período se firmó en 1970 y especifica las áreas a desarrollar: Investigación Nuclear y Desarrollo de la Técnica Nuclear, Oceanografía, Documentación Científica y Procesamiento Electrónico de Datos (MREE, 1970:209-212). 
Los convenios de Cooperación Científico Técnica con la RDA también incluían artículos equivalentes, estableciendo las condiciones de estadía de expertos en Chile, así como becas para formar chilenos en distintas áreas y niveles académicos (MRREE, 1971:333, 337, 344).

Las Memorias del MRREE registran dos acuerdos interuniversitarios. Uno para fomentar el intercambio cultural entre la Universidad de Chile y la Universidad Karl Marx de Leipzig (RDA), firmado en 1964 (AUCH, 1964, Nr. 131:256; MRREE, 1970:217-220), y otro entre la Universidad Georg-August de Göttingen (RFA) y la Universidad Austral de Chile en 1966 (MRREE, 1966:173).

A pesar de la ausencia de vínculos diplomáticos entre Chile y Alemania Democrática, la prensa de ambos países registra una serie de eventos académicos y culturales que ilustran la intensa actividad desarrollada, mencionada también por la literatura secundaria (Möbus, 2005a:36) ${ }^{4}$. Desde 1961 funcionaba en Santiago un Instituto Chileno Alemán Democrático de Cultura, que buscaba ser un contrapeso del Instituto Chileno-Alemán de Cultura, fundado por la RFA en 1952 (Villarroel, 2007; BArch, DY/30/IV A 2/20 726:4)5. El Instituto fue un importante vínculo de ese país con el mundo artístico, político y académico (Emmerling, 2013:99, 104; BArch, DY/30/IV A 2/20 726:116), tenía dos programas radiales (en Radio Magallanes y en la Radio de la Universidad Técnica del Estado) y representaciones en cinco ciudades: Arica, Antofagasta, Valparaíso, Concepción y Temuco (ND, 20.01.1968:6; ES, 05.10.67:4).

En el contexto de la visita de una delegación sindical de la RDA al III Congreso de la Central Única de Trabajadores (1962), el profesor Manfred Kossok de la Universidad Karl Marx de Leipzig realizó varias conferencias, que probablemente fueron el inicio de la larga relación académica que sostuvo con Chile (Emmerling, 2013:76) ${ }^{6}$. Dos años más tarde se firmó entre la Universidad Karl Marx de Leipzig y la Universidad de Chile el ya mencionado acuerdo para promover el intercambio cultural. Como parte de este, el etnólogo Peter Neumann del Museo Etnológico de Dresden realizó una estadía de ocho meses en Chile (NZ, 05.02.1967:2).

En 1966, el rector de la misma Universidad, Georg Müller, visitó Chile (ND, 01.10.66:10; ES, 06.10.66:7) y, en ese mismo año, se firmó un 
acuerdo entre la Universidad Técnica del Estado y la Universidad Técnica de Dresden (ES, 05.10.67:4).

La prensa alemana registra la presencia de académicos chilenos en la RDA. Entre otros, la conferencia del profesor de la Universidad de Chile Carlos Keller, en la Universidad de Rostock en 1963 (BZ, 20.03.1963:1) y la de su Secretario General, Álvaro Bunster, en la Universidad Humboldt de Berlín, el mismo año (ND, 20.02.1963:6), o la visita de una delegación parlamentaria a la Universidad Técnica de Dresden en 1968 (NZ, 28.06.1968:2) ${ }^{7}$.

A lo anterior se debe añadir el trabajo de las Fundaciones Políticas de la RFA en Chile a partir de los años 1960, apoyando o realizando investigaciones (Dufner, 2012) ${ }^{8}$. También es necesario considerar la gran cantidad de proyectos técnicos de la ayuda al desarrollo que la RFA realizaba como complemento al trabajo de Estados Unidos, a través de la Alianza para el Progreso (Dufner, 2012; Villarroel, 2007:94-95).

Para este período sólo se dispone de datos agregados de estudiantes chilenos en la RFA y RDA. La literatura registra 15 chilenos que cursaron doctorados en Ciencias Sociales y Humanidades en el primer país, entre 1954 y 1972, mientras que en la RDA había 51, unos días después del golpe militar (Holtz, 2011). La mayoría de ellos, seguramente, arribados a comienzos de los años 1970. A fines de 1973, había 114 chilenos aprendiendo algún oficio o realizando estudios superiores en la RDA (Emmerling, 2013:404).

Como es posible apreciar, durante este período las relaciones científicas entre Chile y ambas Alemania se ven significativamente influidas por la competencia que libraban la RFA y la RDA por consolidar sus posiciones internacionales, lo que en gran medida explica la alta intensidad que tuvieron. En ellas se aprecia un patrón bastante tradicional de dependencia de las decisiones y financiamientos del "centro", y un rol preponderante del Estado en la conducción de las relaciones académicas. Otro elemento significativo es la integración que existía de la actividad científica, con la cultural y la cooperación técnica, que se articulaba bajo concepto de "ayuda al desarrollo", que constituía una idea central de la política internacional de la época. 


\section{LOS DOS CHILE Y LAS DOS ALEMANIA. DEL GOLPE MILITAR A LA CAÍDA DEL MURO DE BERLÍN}

El siguiente período en las relaciones científicas entre Chile y ambas Alemania transcurrió entre el golpe militar de 1973 y la caída del Muro de Berlín, que coincide, además, con el fin de la dictadura del general Pinochet.

El golpe provocó la ruptura inmediata de las relaciones diplomáticas con la RDA, iniciadas en 1971, y el enfriamiento de los vínculos con la RFA. Desde la perspectiva de la RDA esto significó circunscribir la relación sólo a aspectos comerciales y, en el caso de la RFA, reducir a un mínimo otros tipos de intercambios hasta ese momento habituales.

Ambas Alemania realizaron un amplio trabajo de solidaridad y recibieron un número importante de exiliados, que generaron sus propias organizaciones, varias de las cuales tuvieron reconocimiento formal e incluso contaron con apoyo estatal.

Para Chile, el golpe militar significó una ruptura de su tradición diplomática y un aislamiento internacional sin precedentes, que obligó a la dictadura a realizar un despliegue ante los países en que podría conseguir apoyo y un gran esfuerzo por mantener un vínculo con sus aliados tradicionales.

Durante este período se dieron dos tipos de relaciones científicas: unas en el marco del decaído vínculo con la RFA y, otras, en territorio de ambas Alemania, producto del exilio.

\section{El Chile regido por el general Pinochet y las dos Alemania}

Alemania Federal tuvo ante el régimen de Pinochet una posición ambivalente y pragmática, que la llevó a no romper relaciones con Chile, pero sí a acotarlas.

Por una parte, lo sucedido era considerado positivo por Alemania Federal, ya que resituaba al país dentro del bloque occidental. Pero, por otra, instalaba una dictadura difícilmente compatible con la idea de estado de derecho que subyacía a la política alemana, en especial debido a las violaciones de los derechos humanos e incluso la detención 
de ciudadanos alemanes. Así, mientras la RFA condenaba estos actos ante las Naciones Unidas, pasaba al mismo tiempo a convertirse en el segundo socio comercial de Chile y en el principal mercado para sus exportaciones a Europa (Barrenechea, 1984:5). La RFA apoyó a Chile, además, ante instituciones financieras internacionales para acceder a créditos (Arcos, 1989:111).

En lo que respecta a la cooperación financiera de Alemania Federal, en el período que va desde 1950 a 1982, Chile ocupó el cuarto puesto en América Latina (por debajo de Brasil, Perú y Colombia), mientras que hasta 1968 había estado en el sexto lugar (Dufner, 2012:134).

Con el golpe de Estado, la República Democrática Alemana rompió relaciones de manera casi inmediata con Chile, el 24 de septiembre de 1973, y adoptó una postura más confrontacional (Möbus, 2005a:45). La investigación reciente, sin embargo, ha demostrado que ello no afectó las relaciones comerciales entre ambos países. Al contrario, entre 1973 y 1975 éstas alcanzaron su punto más alto y sólo con posterioridad a 1975, la RDA redujo su intercambio comercial con Chile (Dufner, 2013:541-542). No obstante, en 1976 abrió nuevamente una oficina comercial en Santiago, que culminó en la apertura de una representación comercial en 1980 (Heller, 2012:160).

Chile, por su parte, frente al aislamiento internacional reaccionó liberalizando el comercio y las inversiones en el país, a fin de conseguir capital financiero. Más allá del relativo éxito de esta estrategia, las presiones internacionales por terminar con la dictadura continuaron en aumento, en especial en la década de 1980. Declaraciones y discursos de líderes europeos que recurrentemente ponían énfasis en esta situación eran desacreditados por esta y juzgados como "interferencias en asuntos domésticos" (Portales, 1989:324). Si bien, entonces, no hubo una ausencia de vínculos diplomáticos, sí hubo una pérdida de la capacidad estratégica de utilizarlos en beneficio político propio, como había sucedido hasta ese momento (Fermandois, 1991:441).

Durante todo el período, Chile y la RFA firmaron sólo un acuerdo bilateral, referido a transporte (Arcos, 1989:115). Sin embargo, las Memorias del Ministerio de Relaciones Exteriores registran actividades asociadas al intercambio científico. Además de las menciones ocasionales a la cooperación técnica, es posible identificar actividades entre 
instituciones de educación superior, asignación de becas y otras cooperaciones específicas.

En 1984 y 1985, la ayuda técnica recibida desde distintos países (incluida la RFA) se vinculó con actividades científicas y académicos. En 1984, por ejemplo, estuvo "básicamente orientada al área de la investigación científica” (MRREE, 1984:135), mientras que al año siguiente consistió en un "fuerte apoyo en docencia al sector universitario nacional" (MRREE, 1985:449).

A poco tiempo de iniciada la dictadura, en 1975, se consigna la donación por parte de la RFA de material para la enseñanza del alemán a la Academia Diplomática (MRREE, 1975:1165). Un año más tarde se alude a la prórroga del "Convenio de Cooperación Interuniversitaria" entre las Universidades de Concepción y las de Maburgo y Maguncia, focalizado en química, física y tecnología de polímeros (MRREE, 1976:51). Tanto la fundación del Departamento de Polímeros en 1973, como el doctorado en Ciencias Naturales con especialidad en Química, creado en 1975, contaron con apoyo científico y financiero de la RFA (Rivas, 2009:45).

Con cierta intermitencia y escasa precisión, se informa sobre la asignación de becas por parte de la RFA. Mientras en 1981 sólo se hace mención general al tema, en 1982 se especifica su número (10), así como las postulaciones (18) y asignaciones (1). Al año siguiente se señala la entrega de 15 becas y, para 1986, sólo se menciona la oferta y el uso de una beca (MRREE, 1981:72; 1982:119-120; 1983:188-189; MRREE 1986:293 y ss.).

Hacia fines del período, en 1987, se registra la visita del catedrático alemán de la Universidad de Würzburg, profesor Lothar Bossle (MRREE, 1987:79).

En los dos años siguientes se consigna una actividad que posteriormente se institucionalizará: la colaboración en investigación antártica. En 1988 visitó Chile una comisión de la RFA, con el objetivo de tomar contacto con el Instituto Antártico Chileno. Al año siguiente se realizó una expedición a la Antártica en la que participó la Universidad de Konstanz (MRREE, 1989:162-163). 
A esta actividad es necesario agregar la realizada por las fundaciones políticas alemanas, que tuvo alcances difíciles de cuantificar. A nivel político se conoce y valora el apoyo que dieron a diversas organizaciones y su rol en la renovación programática de los partidos de oposición, en parte gracias a la formación académica entregada a algunos de sus militantes (Dufner, 2013:546; Thesing, 2014:231) ${ }^{9}$.

\section{El Chile expatriado y las dos Alemania}

En la RDA el exilio chileno fue asumido como un asunto de Estado. Con el objeto de coordinar el apoyo a los exiliados se creó en octubre de 1973 un Chile-Zentrum, al amparo del Comité de Solidaridad de la RDA (cuya función era canalizar la ayuda al desarrollo). Al interior del PSUA se fundó además un Grupo de Trabajo Chile (Drechsler, 1978: 296; Emmerling, 2013:380).

Las contrapartes de estas instancias fueron la Comisión de Solidaridad de la Unidad Popular de Berlín, cuya dependencia principal estaba en Roma, y la Oficina Chile Antifascista, dirigida por chilenos, pero reconocida por la administración alemana como interlocutora (Maurin, 2005:365).

Las funciones de estas organizaciones eran preparar la llegada de los exiliados, coordinar el trabajo con organizaciones homólogas de otros países y apoyar actividades de resistencia a la dictadura de Pinochet.

Producto del importante volumen de chilenos que llegó al exilio, fue necesario distribuirlos por toda la RDA, siendo dirigidos en grupos a ciudades donde, además de proveerles vivienda y servicios de distinto tipo, se les podía asignar trabajo (Möbus, 2005b:160).

Se estima que entre 1973 y 1977 llegaron 1.123 exiliados chilenos, y que durante todo el periodo fueron unos 2.000 (Emmerling, 2013:404410; Janert, 2010).

La literatura consigna dos grupos académicos de chilenos que trabajaron en universidades de la RDA. Uno en la Universidad Karl Marx de Leipzig, en el Seminario Latinoamericano, Sección Historia. Creado a fines de 1973 por el PSUA, estuvo a cargo de Manfred Kossock y el cientista político Eberhard Hacketal. Por ese Seminario pasaron varios 
chilenos, desarrollando algunos de ellos estudios de doctorado (Álvarez, 2006:112; Cademartori, 2012:67-69; Möbus, 2005b:164). El otro estaba en la Sección de Latinoamericanística de la Universidad de Rostock, a la que se había integrado en mayo de 1974 (Dessau, 1974:775).

Hacia 1975 había 84 chilenos matriculados (26 en doctorados), en las universidades de Dresden (30), Berlín (18), Leipzig (14), Magdeburg (5) y Rostock (3), en tanto otros estudiaban programas en universidades técnicas. Las disciplinas eran variadas: 62 estudiaban Ciencias Sociales y Humanidades, 11 Medicina, 17 Ciencias Naturales y otros 39 carreras técnicas. Hacia 1977, los matriculados eran 170, de los cuales 27 lo estaban en doctorados. Esto llevó a los chilenos a ser el segundo grupo de latinoamericanos en número de graduados en doctorados (Holtz, 2011:89). En 1980 había 58 alumnos de doctorado chilenos en la RDA (Holtz, 2011:95).

Aunque con una postura más ambivalente, también la RFA aceptó asilar perseguidos desde el inicio de la dictadura, llegando el Estado Federal a un acuerdo con los Bundesländer para distribuirlos. El primer grupo arribó a Frankfurt en diciembre de 1973 y al año siguiente se estima que vivían en Alemania Federal algo más de 2.000 personas. Hasta finales del período superaron los 5.000, transformándose entre 1977 y 1979 en el grupo más grande de latinoamericanos residentes (Dufner, 2012:259-260).

La petición al gobierno federal de recibir exiliados tuvo su origen en actores como sindicatos, iglesias, partidos e incluso instituciones científicas. Además, no menos de ocho delegaciones oficiales viajaron a Chile a intentar conseguir la liberación de prisioneros alemanes y políticos chilenos (Dufner, 2012:255) ${ }^{10}$.

El proceso de instalación en la RFA no fue conducido centralmente pero contó con la coordinación de diversas organizaciones, que ayudaban a los chilenos a solicitar asilo y en su integración (Poutrus, 2015:879). En los meses inmediatos al golpe se formaron en la RFA alrededor de seis comités (Chile Komitees) de apoyo en distintas ciudades y a fines de 1974 llegaban a unos 50 (Balsen y Rössel, 1986:323) A medida que se fue conociendo lo que sucedía en Chile, más actores se integraron al movimiento de solidaridad (Dufner, 2014:164-165; Müller-Plantenberg, 2002b:94). 
No se ha encontrado en la literatura referencias a la formación de núcleos académicos chilenos similares a los de la RDA. Sin embargo, sí se conoce, por ejemplo, la oferta que hizo el Canciller de la Universidad Libre de Berlín de 20 becas de estudio y 3 puestos de trabajo para investigadores, a comienzos del exilio, así como referencias ocasionales a chilenos que trabajaron en universidades e institutos de investigación (Müller-Plantenberg, 2002a:88).

Respecto a los estudiantes de doctorado en la RFA, existen datos agregados para quienes cursaron programas en Ciencias Sociales y Humanidades, entre 1983 y 1992. De los 108 latinoamericanos que se doctoraron en esas áreas, 23 eran chilenos. De ellos, 10 provenían del exilio (Holtz, 2011:121). La mayoría estudió en el Instituto Latinoamericano, de la Universidad Libre de Berlín, o en el Instituto de Ciencias Políticas de la Universidad de Heidelberg. Otros en la Universidad de Erlangen-Nürnberg (Holtz, 2011:178).

Las relaciones científicas durante este período asumieron características distintas a las que habían tenido hasta antes del inicio de la dictadura, debido a la reducción del trabajo y apoyo internacional de la RFA a Chile. La ausencia de acuerdos bilaterales y el retiro del Estado como promotor del intercambio académico, relegó la colaboración a actores individuales y a las fundaciones políticas alemanas. Una mayor intensidad tuvo en territorio alemán, producto de la presencia de exiliados chilenos que se integraron a instituciones académicas, especialmente en la RDA. Este aislamiento que vivió Chile redujo los niveles de dependencia académica, sin embargo, dada la particular situación del país, no se generó como reacción un desarrollo científico endógeno significativo, sino que la actividad científica en general quedó relegada a un segundo plano.

\section{CHILE Y ALEMANIA: EL RETORNO A LA TRADICIÓN Y LA COMPETENCIA GLOBAL POR EL CONOCIMIENTO}

La caída del muro de Berlín y la reunificación alemana, por una parte, y el fin de la dictadura de Pinochet, por la otra, marcaron la normalización de las relaciones bilaterales y científicas entre Chile y Alemania.

El proceso de reunificación obligó a Alemania a redefinir su política exterior, tanto por su nueva realidad, como por la necesidad de pensar en una posición internacional sin la presión del alineamiento en torno a dos grandes bloques. Esto implicaba, al menos, reconsiderar su situa- 
ción en Europa, su rol en el proceso de integración europea y redefinir su papel en la OTAN y en los conflictos con que esta organización debió lidiar durante la década del 90 (Schöllgen, 2013:158 y ss).

A Chile, el regreso a la democracia le permitió restaurar su inserción diplomática. Esto implicó recomponer sus relaciones con los países vecinos y encaminarse a la apertura a mercados internacionales, a través de la firma de unos 20 tratados de libre comercio con varios países. Entre ellos: Estados Unidos (2003), Canadá (1996), la Unión Europea (2002) y países de Asia Pacífico (van Klaveren, 2011:163).

\section{La internacionalización de la educación superior y ciencia}

Las relaciones científicas entre Chile y Alemania se daban ahora en un contexto que experimentaba significativos cambios. Por una parte, el fin de la Guerra Fría provocó el surgimiento de otros actores internacionalmente relevantes, por otra, el conocimiento se estaba transformando en un factor decisivo del desarrollo económico y, por último, los sistemas de educación superior y ciencia estaban sufriendo acelerados procesos de expansión y consolidación (Schütte, 2008:12-13).

Evidentemente, no es el objetivo detallar toda esa evolución, por lo que las líneas que siguen analizan el aspecto más relevante para el propósito de este artículo: el surgimiento de las ideas y políticas de internacionalización de la educación superior y ciencia en ambos países, que provocarán una intensificación hasta ahora desconocida de las relaciones científicas internacionales.

El concepto de internacionalización, junto a los de educación de élite, evaluación y aseguramiento de la calidad, ha dominado el debate alemán desde la segunda mitad de la década de 1990. Con este, se alude a que las universidades y la ciencia alemanas deberían aumentar su competitividad internacional, a la vez que Alemania debería volverse un lugar atractivo para estudiantes y científicos extranjeros (Fuchs y Reuter, 2000:101-103). Este debate se insertaba en el contexto europeo, que desde 1987 desarrollaba programas de movilidad estudiantil y, a partir de 1999, el conocido proceso de Bolonia (Matus, 2015:449).

Ya en el año 1992 el Consejo de Ciencia había elaborado un documento con "Recomendaciones para Internacionalizar las Relaciones Cien- 
tíficas" (Wissenschaftsrat, 2010:5) y, en 1996, la Conferencia de Ministros de Cultura editó una declaración para hacer más atractiva Alemania para los estudiantes internacionales (Alesi y Kehm, 2010:47).

Un punto culminante fue la promulgación de la Ley Marco de Educación Superior en 1999, que estableció una nueva forma de relación entre el Estado y las instituciones. Esta ley, aumentó la desregulación con el propósito de intentar hacer más eficiente el uso de los recursos y dar mayor autonomía a las instituciones, entre otros aspectos (Fuchs y Reuter, 2000:101-103) ${ }^{12}$.

La institucionalidad alemana respondió a la política de internacionalización de diversas maneras. El DAAD propuso en 1997 unas Estrategias Internacionalización que incluían la oferta de programas de bachillerato y maestría en inglés a fin de atraer estudiantes extranjeros (Fuchs y Reuter, 2000:113). El programa inicial del DAAD fue complementado con otros en los años venideros: 2000, 2004 y 2008 (Alesi y Kehm, 2010:48) y estuvo acompañado de iniciativas destinadas a promocionar a las instituciones de educación alemanas en el extranjero.

También la Fundación Alexander von Humboldt se hizo cargo del tema, organizando en 2007 un encuentro entre más de 30 expertos, que dio pie a un documento de trabajo titulado Política Científica Exterior - Politica Exterior de Ciencia (AvHSt, 2007). Lo mismo la Sociedad Alemana de Ciencia (DGF), que en 2012 editó su Estrategia de Internacionalización (www.dfg.de).

Paralelo a estas estrategias y actividades se desarrolló una larga lista de instrumentos de política pública para fomentar el intercambio científico. Entre ellos se debe destacar la Iniciativa de Excelencia iniciada en el 2005, que buscaba visibilizar y hacer más competitivas las universidades alemanas (www.bmbf.de).

Esta discusión se dio, además, en el marco de otra mayor, a nivel europeo, que culminó con la creación del Consejo Europeo de Investigación en el año 2007 (Winnacker, 2008:88-89).

A mediados de la década del 2000, la idea de "Política Científica Internacional", se había convertido en un concepto más de las políticas 
científica y exterior alemanas, lo que se expresa en el documento programático elaborado por el Ministerio Federal de Educación y Ciencia en 2008, que definía una estrategia de internacionalización para la ciencia alemana (Alesi y Kehm, 2010:52; BMBF, 2008; Schütte, 2008:24).

Chile, por su parte, durante toda la primera mitad de la década de 1990 debió abocarse a reconstruir y fortalecer su sistema de ciencia y tecnología, cuyo crecimiento se había prácticamente estancado en el período anterior. Desde el Ministerio de Educación se buscó por una parte, aumentar las capacidades docentes de las universidades y, por otra, comenzó una paulatina diversificación y crecimiento de los instrumentos y recursos para la actividad científica (Fernández, 2015).

Hacia mediados de la década de 1990, Chile se hizo cargo del tema de la internacionalización integrándolo como un eje del Programa de Mejoramiento de la Calidad de la Educación Superior (Mecesup), en 1998. Sin embargo, a pesar de las referencias a la internacionalización en los programas de gobierno desde el presidente Frei en adelante, no se ha elaborado un plan detallado para lograr dicho objetivo (OCDE, 2009:157).

Su fomento se ha dado, principalmente, por iniciativas que las distintas agencias e instituciones de educación superior han llevado a cabo. En el contexto del Programa Mecesup se desarrollaron actividades destinadas a fomentar la movilidad estudiantil y la vinculación internacional de los doctorados; la Comisión Nacional de Ciencia y Tecnología (Conicyt), implementó programas para apoyar la formación de redes supranacionales y los estudios de postgrado de chilenos en el extranjero; también firmó acuerdos con distintos países, a fin de aumentar la movilidad estudiantil y académica. Organizaciones como el Consejo de Rectores han firmado convenios con organizaciones homólogas de Alemania, Bélgica, Brasil, Canadá, España y otros, destinados a favorecer el intercambio estudiantil, desarrollar programas de postgrado comunes y facilitar el intercambio científico (www. mecesup.cl; www.conicyt.cl; Caiceo, 2010:14 y ss).

$\mathrm{Al}$ igual que en Alemania, la discusión nacional se dio en el contexto del proceso de Bolonia y en el marco de iniciativas regionales de internacionalización, como el Programa Tuning, y otras supra regionales, que buscan formar un espacio común de educación superior que incluyera la Unión Europea, América Latina y el Caribe (Matus, 2015:450). 
A pesar de los desequilibrios que existen en el grado de integración internacional del sistema de educación superior y ciencia chilenos, producto de la ausencia de un plan nacional y de las distintas prioridades que el tema tiene en las agendas institucionales (OCDE, 2009:157), este se ha orientado cada vez más hacia su entorno internacional y exhibe importantes logros (Caiceo, 2010:14 y ss; Geldres et al., 2013; Conicyt, 2014).

\section{Las nuevas dinámicas de las relaciones científicas chileno-alemanas}

Hasta mediados de la década de 1990 las relaciones científicas chileno-alemanas tuvieron un ritmo bajo y descansaron en la iniciativa individual de investigadores e instituciones que ya contaban con inserción y tradición de trabajo internacional (Rojas, 2010:10). Esto cambió a medida que avanzaba la década.

Entre el año 1991 y 2014, Alemania y Chile firmaron 89 acuerdos bilaterales, pero sólo uno se refiere a "Educación, Ciencia, Tecnología e Innovación", los restantes corresponden a cooperación técnica, financiera, acuerdos comerciales y diplomáticos (www.leychile.cl) ${ }^{13}$. Dicho acuerdo, promulgado en septiembre de 2013, establece un marco general para fomentar el intercambio académico y científico. Correspondientemente, define las áreas del conocimiento en que se aplicará (www.leychile.cl).

La situación descrita, sin embargo, no representa la dinámica que han tomado las relaciones científicas chileno-alemanas, sino que expresa el nuevo rol del Estado en ellas.

Un primer elemento interesante del período actual es que la firma de los convenios ha sido coordinada y realizada a nivel de ministerios, fundaciones y agencias de gobierno a cargo del desarrollo científico y tecnológico. Esto da cuenta de la consolidación de la institucionalidad de ambos países en esta área.

En marzo del 2009 se firmó un convenio marco entre el Servicio Alemán de Intercambio Académico (DAAD) y Conicyt, con el propósito de profundizar las relaciones existentes. Dicho convenio es la culminación de un proceso que se inició con un primer acuerdo en 1998. Otros similares se han firmado entre Conicyt y la Sociedad Alemana 
de Ciencia (DFG) en el 2002, con la Sociedad Max Planck (MPG) en 2008 y con la Fundación Alexander von Humboldt en 2009 (Rojas, 2009:10; www.conicyt.cl; www.bmwf.de).

La Conferencia de Rectores de las Universidades Alemanas (HRK) estableció contacto a mediados de la década del 90 con su par chileno, el Consejo de Rectores, desarrollando una serie de reuniones bilaterales. Ellas derivaron en 1999 en la firma de un primer convenio y en 2009 publicaron una declaración para la creación de programas de doctorados conjuntos (Bongers, 2009:59; www.hrk.de).

Un segundo elemento relevante es que la mayor autonomía de las instituciones y su esfuerzo por internacionalizarse han llevado a una gran proliferación de convenios interinstitucionales. En 2009, el director del DAAD en Chile cifraba su número en alrededor de 100 (Bongers, 2009:59). Al año 2015, éste había llegado a 264 (www.hochschulkompass.de). Estos datos reflejan la dinámica que ha tomado la firma de acuerdos entre universidades chilenas y alemanas.

Una nueva dimensión que trasciende el clásico intercambio científico centrado en la investigación y la docencia, es la creación conjunta de programas de doctorado. A fines de 2015 existían cuatro: Agronegocios, Astrofísica, Sicología y Biología (www.hrk.de).

El intercambio de alumnos también ha tenido una evolución interesante y significativa. Si bien los estudiantes latinoamericanos en Alemania han aumentado, estos han perdido importancia en relación a los de otras regiones. En 1983 y 1984 estudiaron 2.898 latinoamericanos en la RFA y entre 1993 y 1994 llegaron a 4.635. Desde ahí en adelante esa cifra comenzó a estancarse: en 1996 y 1997 fueron 4.693. En el mismo período se triplicaron los estudiantes procedentes del África Subsahariana (llegando a 14.462 en 1996 y 1997) y se produjo una "explosión de estudiantes provenientes de los Países de Europa Central y Oriental" (Hofmeister, 1998:68).

Los estudiantes de doctorado chilenos en Alemania siguen un patrón similar, en relación a otros países latinoamericanos. Los datos del DAAD para el período 1994-2014, muestran que mientras los beneficiarios totales de Chile pasaron de 136 a 467, los latinoamericanos lo hicieron de 1.442 a 8.410. Más decidor es que luego del impulso dado 
a los becarios en 2006, que permitió superar los 450 anuales, este número se estancó, en tanto que el de latinoamericanos sigue creciendo (www.daad.de). Es necesario señalar, sin embargo, que Chile continúa ocupando entre el tercero y cuarto lugar, luego de México, Brasil y Argentina.

En lo que respecta a las becas para estudios en el extranjero se aprecia que, de las 1.832 que el gobierno chileno entregó entre 1990 y 2006, sólo 38 fueron a Alemania, y en cinco de esos 17 años ningún chileno estudió por este medio en ese país (Bodemer, 2009:12-13).

Para el período 2008-2014, el número de beneficiarios del programa de doctorados Becas Chile que eligió Alemania fue de 129, ocupando la sexta preferencia, siendo ampliamente superada por Reino Unido (518), Estados Unidos (479) y España (474), y ligeramente por Australia (165) y Francia (146) (www.conicyt.cl). Esos 129 estudiantes representan el 5,6\% del total de los becarios.

Para el mismo período, el número de estudiantes chilenos adscritos al programa de magíster de Becas Chile que escogió Alemania fue sólo de 55, representando un 1,8\% de los 3.035 que fueron al extranjero (www.conicyt.cl).

Por último, es importante destacar una nueva forma de relación científica surgida: la instalación de instituciones alemanas (y de otros países) en Chile. La primera de ellas llegó en el 2002 a Santiago y corresponde a una sede de la Universidad de Heidelberg que ofrece estudios de postgrado y postítulo: el Heidelberg Center para América Latina (www.bmwf.de; www.heidelberg-center.uni-hd.de).

En el año 2010 arribó la Sociedad Fraunhofer, dedicada a la investigación aplicada, instalando dos Centros de Excelencia: uno en biotecnología de sistemas y otro (fundado en 2015), para generar tecnologías para la energía solar (www.bmwf.de; www.fraunhofer.cl). De igual modo, la Sociedad Max Planck ha apoyado al Centro Interdisciplinario de Neurociencias de la Universidad de Valparaíso, fundado en 2009 (www.santiago.diplo.de; www.cinv.uv.cl).

Esta nueva forma de relación científica da cuenta de los actuales conceptos y estrategias de internacionalización. Debido a la relevancia 
que la ciencia ha tomado en el desarrollo económico, los países se han orientado hacia la búsqueda de estudiantes, académicos y conocimiento de punta, con independencia del lugar en que se encuentren.

Donde mejor se aprecia esta estrategia es en aquellas áreas en que contando con tecnología de vanguardia - no se dispone en el propio territorio de una industria que demande avances en dicha tecnología, con el consiguiente riesgo de no poder seguir generando desarrollos relevantes para la industria. En el caso de Alemania, esta situación se vive en la industria minera y es en una medida importante, la que la ha llevado a instalar dependencias de la Sociedad Fraunhofer en Chile (Schories, 2009:20).

En este tercer período que se inicia con los años 1990, las relaciones científicas se han retomado entre los dos actores existentes: Chile y Alemania. Sin embargo, el rol de Estado ha cambiado, pasando de liderar los procesos de intercambio a definir marcos normativos que los hagan posibles y los fomenten. Esto, junto al nuevo contexto internacional, ha provocado, por una parte, un aumento de la intensidad en las relaciones y, por otra, una expansión de estas hacia nuevos actores científicos relevantes. Dicha situación es una señal inequívoca que la dependencia académica en su sentido más clásico ha comenzado a cambiar. No obstante, existen indicios claros que lejos de desaparecer, esta parece estar focalizándose en las áreas de interés estratégico de países del centro, como se aprecia en la llegada de instituciones de investigación alemanas a Chile.

En otras palabras, las relaciones científicas internacionales se han vuelto un componente central de la política internacional contemporánea, pero ya no como un instrumento más de una lucha ideológica, sino como un factor decisivo para asegurar la prosperidad de los países. Es en este contexto, entonces, en el que las relaciones científicas chileno-alemanas están teniendo lugar y deberán desarrollarse en el futuro.

\section{CONCLUSIONES}

Como se ha podido apreciar, las relaciones científicas chilenoalemanas a partir de la segunda mitad del siglo XX han seguido patrones similares a los descritos por la literatura relativa a la depen- 
dencia académica y cultural. Es decir, han estado significativamente influidas por decisiones que se han tomado en el centro (tanto en la RFA como en la RDA) y que han definido no sólo la intensidad del intercambio, sino también aspectos centrales de su contenido.

Existen en el período, sin embargo, algunas especificidades, cuyo análisis permite caracterizar de modo más preciso estas relaciones, identificando etapas, actores y ámbitos, además del rol que en ellas han jugado el contexto internacional, las circunstancias políticas de cada país y el desarrollo de sus propios sistemas de educación superior y ciencia.

La primera etapa identificable transcurrió entre el fin de la Segunda Guerra Mundial y septiembre de 1973, en que se produjo el golpe de estado en Chile. En ella, las relaciones científicas se dieron entre un Chile y las dos Alemania: la República Federal y la República Democrática. Mientras con la RFA se articularon en un marco diplomático, con la RDA - hasta 1971 - los principales protagonistas del intercambio no fueron el Estado y sus agencias, sino organizaciones como partidos políticos, agrupaciones sociales e instituciones de educación superior. La competencia entre ambas Alemania - intensificada por el giro a la izquierda de la política chilena - definió en gran medida la alta intensidad que tuvo el intercambio, ya que las relaciones científicas eran parte de un concepto mayor asociado a la propaganda cultural, que ambos bloques ideológicos realizaban globalmente. Los Estados jugaron en esta etapa un rol preponderante en el desarrollo de las relaciones científicas y ellas tuvieron una fuerte imbricación con la cooperación técnica y la actividad cultural, que eran en parte utilizados como instrumentos de una política exterior que buscaba consolidar posiciones ideológicas antagónicas. En esta etapa se reproducen patrones conocidos, bastante asimétricos en la relación científica, ya que las decisiones del centro (ambas Alemania) influían decisivamente las condiciones de la colaboración. Todo ello se veía intensificado por la baja estructuración y autonomía de los sistemas de ciencia y tecnología, particularmente en Chile.

El acuerdo de reconocimiento mutuo entre ambas Alemania (1972) y la dictadura en Chile (1973) marcan el inicio del segundo período en las relaciones científicas. Este momento se caracterizó por el fin de la competencia entre la RFA y la RDA, por la ruptura de las relaciones diplomáticas con el segundo país y por el enfriamiento de éstas con 
Alemania Federal, la cual redujo su actividad con Chile al mínimo, delegándola en diversas agencias y en contactos institucionales e individuales. Durante este período, otro Chile se agregó al regido por el general Pinochet: el expatriado, formado por los exiliados chilenos en el extranjero. Con él, ambas Alemania se relacionaron de manera diferente. Mientras la RFA consideró a los exiliados como ciudadanos individuales, la RDA, hizo de éste un asunto de Estado, reconociendo sus organizaciones como interlocutoras y promoviendo su inserción laboral, también en el campo académico y científico. Paradójicamente, la ruptura de relaciones diplomáticas entre Chile y la RDA no implicó una extinción de sus vínculos científicos, sino su intensificación.

La caída del muro de Berlín y el fin de la dictadura en Chile dan inicio al tercer período en las relaciones científicas, que se retomaron sólo entre los dos actores actuales, en el contexto de la diplomacia formal ${ }^{14}$. Esta etapa, sin embargo, se dio en un contexto internacional y nacional significativamente distinto a las anteriores. Por una parte, ante la consolidación y complejización de los sistemas nacionales de educación superior y ciencia, los Estados ya no podrán conducir centralmente estas relaciones, como lo habían hecho antes, sino que se abocaron a definir marcos normativos y a construir instrumentos de política que las hicieran viables. Por otra parte, las relaciones científicas adquirieron una dimensión internacional nueva, acorde a la realidad de un mundo multipolar, en el cual el conocimiento ha adquirido un valor estratégico y las posibilidades de relacionarse científicamente han crecido de una manera hasta ahora desconocida. Por último, la esfera científica se ha escindido de la cooperación técnica y del trabajo cultural, adquiriendo una dinámica de desarrollo autónoma. Efectivamente en ciertos convenios mantienen algún grado de interrelación, pero se trata de aquellos cuya ejecución requiere de apoyo científico.

El nuevo contexto internacional ha generado una enorme diversificación de las oportunidades para construir otras alianzas o competir con actores que han emergido recientemente. Producto de ello, las relaciones científicas de ambos países se han hecho más internacionales y se han orientado en múltiples direcciones. Una consecuencia ha sido que, pese a la recuperación de las relaciones diplomáticas y de la creciente firma de acuerdos interinstitucionales, el número de chilenos que escoge Alemania para cursar estudios se ha estancado, perdiendo espacio frente a sus pares latinoamericanos. 
Este mayor grado de autonomía de la esfera científica no se ha alcanzado, sin embargo, respecto de la política exterior. Al contrario, se puede afirmar que como nunca antes las relaciones científicas internacionales se han vuelto parte fundamental de ella. Sólo que desde una perspectiva y lógica nuevas. Primero, porque ya no constituyen una herramienta más al servicio de una disputa ideológica bipolar, sino un elemento fundamental para consolidar posiciones en un mundo en el cual el conocimiento ha pasado a jugar un rol decisivo. Por lo mismo, muchas relaciones se han fortalecido, especialmente en las áreas de interés estratégico para Alemania. Segundo, porque se ha comprendido que su mayor efectividad no se conseguirá conduciéndolas centralmente, sino generando los espacios para que estas se desplieguen, aún a riesgo de que ello no ocurra con los socios históricos.

En este escenario es esperable que la colaboración científica en muchos campos disciplinarios se haga más diversa y vaya más allá de las tradicionales relaciones de dependencia académica Norte-Sur. En otros, de interés estratégico para los países del centro, es probable que a pesar del aumento de la competencia global por el conocimiento esto no suceda y ese tipo de relaciones se tiendan a fortalecer.

(Recebido para publicação em 29 de abril de 2016)

(Reapresentado em 25 de abril de 2017)

(Aprovado para publicação em 24 de março de 2019)

\section{NOTAS}

1. Un antecedente es el trabajo de Loeber (2008), Naturwissenschaftliche Forschung in Deutschland in Zusammenhang mit der chilenen Salpeter Wirtschaft. München: GRIN Verlag $\mathrm{GmbH}$.

2. La representación de la RDA fue reabierta en 1966.

3. México votó en contra de la expulsión.

4. Esta actividad correspondía a lo que se denominaba propaganda cultural, y era desarrollada por los países de los bloques opuestos para fortalecer su imagen internacional.

5. No existe precisión sobre la fecha de fundación, pero hay indicios que ya en 1959 había iniciado actividades (Emmerling, 2013:95).

6. La prensa alemana registra también conferencias del profesor Kossok en Chile al año siguiente (NZ, 01.10.1963:4). 
7. A nivel artístico y cultural también hubo una actividad muy intensa. Entre los artistas nacionales que visitaron la RDA se cuentan Violeta Parra en 1962 (BZ 01.091962:8), el Conjunto Folclórico de la Universidad de Chile en 1965 (ND 03.02.1965:4), Silvia Infanta y los Cóndores en 1966 (ES 13.11.66:22) y el pianista Óscar Gacitúa en el mismo año (ES 31.01.67:9). También visitaron la RDA Pablo Neruda y Francisco Coloane, en 1965 (Schidlowsky, 2008:1131-1132; BZ 22.05.65:3). Un número igualmente importante de artistas de Alemania Democrática visitó Chile en este período.

8. Un rol preponderante lo tuvo la Fundación de la Democracia Cristiana, Konrad Adenauer, que llegó a Chile en 1964. Entre sus integrantes se encontraban quienes posteriormente serían destacados expertos en temas de Chile y Latinoamérica: Dieter Nohlen, Franz-Josef Hinkelammert y Norbert Lechner (Dufner, 2012:107).

9. Varios de ellos estudiaron con Dieter Nohlen, que en los años 60 había trabajado en la Fundación Konrad Adenauer en Santiago. Entre 1972 y 1983 se doctoraron con él 4 de los 5 chilenos que estudiaron en Heidelberg becados por la Fundación (Holtz, 2011:179).

10. Entre los detenidos estaba el sociólogo Klaus Meschkat, quien en marzo de 1973 se integró a la Universidad de Concepción. Luego de su liberación asumió una cátedra en la Universidad de Hannover (Holtz, 2011:180-181).

11. Su fruición se debió también a las divisiones entre distintas facciones políticas (Balsen y Rössel, 1986:325).

12. La anterior Ley Marco de 1976 ya consideraba la internacionalización, sin embargo, la nueva versión generó condiciones para que las instituciones pudieran desarrollarla activamente.

13. Los acuerdos de cooperación técnica contienen, en general, un componente científico, expresado en el envío de expertos alemanes a Chile y en la capacitación de chilenos en Alemania (www.leychile.cl).

14. En 1989 hubo un Cambio de Notas entre Chile y la RDA, en el que acordaron reanudar relaciones consulares a partir del año siguiente (MRREE, 1989:185-186). En marzo de 1990 se firmó el protocolo para el restablecimiento de relaciones diplomáticas e, incluso, un acuerdo que creaba un "fondo complementario para estudios y expertos" (MRREE, 1990:260). 


\section{Enrique Fernández Darraz}

\section{BIBLIOGRAFÍA}

ALARCÓN, Cristina. (2005), La génesis de la formación docente inicial de enseñanza secundaria en Chile: un estudio socio-histórico sobre la influencia alemana en el discurso pedagógico fundacional de docentes secundarios, 1889-1910: el caso del Instituto Pedagógico. Tesis (Maestría en Ciencias Sociales con orientación en Educación), FLACSO, Buenos Aires.

. (2010), El discurso pedagógico fundacional de docentes secundarios. Sobre la transferencia educativa alemana en Chile (1889-1910). Buenos Aires: FLACSO.

. (2014), Modelltransfer im Schatten des Krieges. "Deutsche" Bildungs- und "Preußische" Militärreformen in Chile, 1879-1920. Frankfurt am Main: Peter Lang Edition.

ALATAS, Syed. (2000), "Academic dependency in the social sciences: relefections on India and Malaysia". American Studies International, v. XXXVIII, n. 2, pp. 80-96.

(2003), "Academic dependency and the global division of the labour in the social sciences". Current Sociology v. 51, n. 6, pp. 599-613.

ALESI, Bettina; KEHM, Barbara. (2010), "Internationalisierung von Hochschule und Forschung". [Electronic ed.]. Edited by Hans-Böckler-Stiftung. Hans-Böckler-Stiftung. Düsseldorf (Arbeitspapier, 209).

ÁLVAREZ, Rolando. (2006), “¿La noche del exilio? Los orígenes de la rebelión popular en el Partido Comunista de Chile". In: V. Valdivia; R. Álvarez; J. Pinto (eds.), Su revolución contra nuestra revolución. Izquierdas y derechas en el Chile de Pinochet (1973-1981). Santiago: LOM, pp. 101-152.

ARCOS, Enrique. (1989), Política Exterior de Chile hacia la República Federal Alemana. Tesis (Magíster en Estudios Internacionales). Universidad de Chile, Santiago de Chile.

AvHSt. (2007), Außenwissenschaftpolitik - Wissenschaftaußenpolitik. Stiftung Wissenschaft und Politik. Alexander von Humboldt Stiftung. Bonn (Arbeits- un Diskussionspapier 7).

BALSEN, Werner; RÖSSEL, Karl. (1986), Hoch die internationale Solidarität. Köln: Kölner Volksblatt Verlag.

BARRENECHEA, Carlos. (1984), Bundesrepublik und Chile. Die politischen und wirtschaftlichen Beziehungen der Bundesrepublik Deutschland zur Regierung Chile während der Regierungen Frei, Allende und Pinochet. Köln: Pahl-Rugenstein Verlag.

BEIGEL, Fernanda (2013a): “Centros y periferias en la circulación internacional del conocimiento". Nueva Sociedad, n. 245, pp. 110-123.

(2013b), David y Goliath. "El sistema académico mundial y las perspectivas del conocimiento producido en la periferia". Pensamiento Universitario, v. 15, n. 5, pp. 15-34.

(2016), “El nuevo carácter de la dependencia intelectual”. Cuestiones de Sociología, n. 14 , pp. $1-17$

BIRLE, Peter; FERNÁNDEZ, Enrique. (2003), Miradas Alemanas. Chile en las Ciencias Sociales Alemanas 1970-2001. Concepción: Ediciones Escaparate. 
BLUMENAU, Berhard. (2011), "The Map of Africa. Lies in Germany: The Two Germanys and their Struggles for Recognition in Africa". Working Papers in International History, n. 10. Geneva, Graduate Institut of International and Development Studies.

BMBF (Bundesministerium für Bildung und Forschung). (2008), Deutschlands Rolle in der globalen Wissensgesellschaft stärken. Strategie der Bundesregierung zur Internationalisierung von Wissenschaft und Forschung. Berlin-Bonn: BMBF.

BODEMER, Klaus. (2009), "Die deutsch-chilenische Wissenschaftskooperation - keine Sonderrolle aber wichtige Impulse für Neubelebung". In: A. von H. Stiftung (eds.), Deutsch-Chilenische Forschungszusammenarbeit: Tradition und Zukunft. Bonn (Diskussionspapiere, 14), pp. 12-17.

BONGERS, Wolfgang. (2009), “Der Deutsche Akademische Austausch Dienst (DAAD) in Chile: Kooperationen und Projektionen". In: A. von H. Stiftung (eds.), Deutsch-Chilenische Forschungszusammenarbeit: Tradition und Zukunft. Bonn (Diskussionspapiere, 14), pp. 57-61.

CADEMARTORI, José. (2012), Memorias del Exilio. Santiago: Editorial Usach.

CAICEO, Jaime. (2010), "Educación Superior en Chile y su Internacionalización". In Revista HISTEDBR [on-line]. n. 38, [29/3/16] pp. 12-23. http://www.histedbr.fe.unicamp.br/ revista/edicoes/38/art02_38.pdf. ISSN: 1676-2584. Acesso em 29 de fevereiro de 2016.

CONICYT (Comisión Nacional de Ciencia y Tecnología). (2014), “Principales indicadores cienciométricos de la actividad cientifica chilena 2012". In: CONICYT, Informe 2014: una mirada a 10 año. Madrid - Valparaíso: CONICYT.

COSTA, Sérgio. (2014), "Social sciences and north-south-asymmetries: towards a global sociology". En: Sabine Broeck y Carsten Juncker (eds.), Postcoloniality - Decoloniality black critique. Joints and fissures. Frankfurt / New York: Campus, pp. 231-243.

DAAD (Deutscher Akademischer Austauschdienst). (2007), Abschlussbericht: Projekt Alumni Andenländer. Ecuador, Paraguay, Peru, Uruguay und Venezuela. Bonn: Prospecta-DAAD.

DESSAU, Adalbert. (1974), "Die Sektion Lateinamerikawissenschaften der Universität Rostock im 25. Jahr der Deutschen Demokratischen Republik". Asien, Afrika, Lateinamerika, v. 2, n. 5, pp. 767-777.

DRECHSLER, Horst. (1978), "Die politischen, wissenschaftlichen und kulturellen Beziehungen der DDR zu den Ländern Lateinamerikas". Asien, Afrika, Lateinamerika 6 (2), pp. 283-303.

DUFNER, Georg. (2007), Chile als Bestandteil des revolutionären Weltprozesses. Die Chilepolitik der DDR im Spannungsfeld von außenpolitischen, ökonomischen und ideologischen Interessen 1952-1973. Tesis (Magíster Artium). Universidad de Humboldt, Berlin.

. (2012), Chiles Entwicklung, der Kalte Krieg und politische Experimente. Beziehungen in Politik und Wirtschaft zwischen der Bundesrepublik Deutschland und Chile, 1949 bis 1980. Tesis (Doctorado de Historia). Universidad Libre de Berlín, Berlin.

. (2013), "Chile als Partner, Exempel und Prüfstein". Vierteljahrshefte für Zeitgeschichte v. 61, n. 4 , pp. 513-549. 


\section{Enrique Fernández Darraz}

DUFNER, Georg. (2014), “West Germany: Professions of Political Faith, the Solidarity and New Left Imaginaries”. In: K. Christiaens; I. Goddeeris; M. Rodriguez-Garcia (eds.), European solidarity with Chile, 1970s-1980s. Frankfurt am Main, Peter Lang Edition, pp. 163-186.

DUSSEL, Enrique; FORNAZZARI, Alessandro. (2002), "World system and "trans"-modernity". Nepantla: Views from the South, v. 3, n. 2, pp. 221-244.

EMMERLING, Inga. (2013), Die DDR und Chile (1960-1989). Aussenpolitik, Aussenhandel und Solidarität. Berlin: C. H. Links.

FERMANDOIS, Joaquín. (1991), “De una inserción a otra: Política exterior de Chile, 19661991". Estudios Internacionales, v. 24, n. 96, pp. 433-455.

FERNÁNDEZ, Enrique. (2015), "Políticas Públicas de Educación Superior desde 1990 hasta el presente". In: A. Bernasconi (ed.), La educación superior en Chile. Transformación, desarrollo y crisis. Santiago: Ediciones Universidad Católica de Chile, pp. 173-217.

FUCHS, Hans-Werner; REUTER, Lutz-Rainer. (2000), Bildungspolitik in Deutschland. Entwicklungen, Probleme, Reformbedarf. Opladen: Leske + Budrich.

GELDRES, Valeska; VÁSQUEZ, Fabiola; RAMOS, Heidy. (2013), “Internacionalización de la educación superior en Chile. Movilidad internacional de estudiantes en la Universidad de la Frontera". Revista CTS, v. 8, n. 24, pp. 47-64.

GUTIÉRREZ, Claudio; GUTIÉRREZ, Flavio. (2004), “Carlos Grandjot, tres décadas de matemáticas en Chile: 1930-1960". Boletín de la Asociación Matemática Venezolana 1, v. XI, pp. 55-84.

HELLER, Friedrich Paul. (2012), Pinochet. Eine Täterbiografie in Chile. Stuttgart: Schmetterlingverlag.

HERRERA, Amilcar. (1991), “Ciencia y Tecnología en América Latina”. In: N. Cairo; F. Ferrán; C. Cuello (eds.), Quehacer Científico I. Lecturas. Santo Domingo: INTEC, pp. 91-110.

HOFMEISTER, Wilhelm. (1998), “Alemania y América Latina. ¿Relaciones sin emociones?”. Estudios internacionales, v. 31, n. 121-122, pp. 55-71.

HOLTZ, Menja. (2011), Wissenschaftsaustausch als hierarchisierter Transfer. Lateinamerikanische Promotionen in Deutschland. Baden Baden: Nomos.

JANERT, Josefine. (2010), "Das war wie ein zweites Exil. Die Geschichten der Emigranten interessieren in Chile kaum jemand". Neues Deutschland, 1/19/2010. https://www. neues-deutschland.de/artikel/163303.das-war-wie-ein-zweites-exil.html

JÖNS, Heike. (2002), Grenzüberschreitende Mobilität und Kooperation in den Wissenschaften. Deutschlandaufenhalte US-amerikanischer Humboldt-forschungspreisträger aus einer erweiterten Akteurnetzwerkperspektive. Heidelberg, Ruprecht-Karls-Universität Heidelberg (Dissertation).

(2009), “'Brain circulation' and transnational knowledge networks: studying long-term effects of academic mobility to Germany, 1954-2000". Global Networks v. 9, n. 3, pp. 315-338.

KAUFMAN, Edy. (1976), "La política exterior de la Unidad Popular Chilena”. Foro internacional, v. 17 n. 2, pp. 244-274. 
KEIM, Wiebke (2008): "Social sciences internationally: The problem of msarginalisation amd its conequences for the discipline of sociology". En: African Sociological Review, CODESERIA, v. 12 , n. 2, pp. 22-48.

KILIAN, Werner. (2001), Die Hallstein-Doktrin. Der diplomatische Krieg zwischen der BRD und der DDR 1955-1973: aus den Akten der beiden deutschen Aussenministerien. Berlin, Duncker \& Humblot.

KRÄMER, Raimund. (2011), “De una diplomacia desaparecida. La política exterior de la República Democrática Alemana y sus relaciones con América Latina". Estudios internacionales, v. 28, n. 110, pp. 174-197.

LENGWILER, Martin. (2010), "Kontinuität und Umbrüche in der deutschen Wissenschaftspolitik des 20. Jahrhunderts". In: D. Simon; A. Knie; S. Hornbostel (eds.), Handbuch Wissenschaftspolitik. Wiesbaden: VS Verlag für Sozialwissenschaften / GWV Fachverlage $\mathrm{GmbH}$, pp. 13-25.

LOEBER, Katharina. (2009), Naturwissenschaftliche Forschung in Deutschland in Zusammenhang mit der chilenen Salpeter Wirtschaft. München: GRIN Verlag GmbH.

MÄHLERT, Ulrich. (2009), Kleine Geschichte der DDR. München: Beck.

MARTÍNEZ, Luis. (2011), “Colonialidad del poder: el grillete de nuestra historia". Temas, n. 65 , pp. $4-13$.

MATUS, Claudia. (2015), “Discursos de internacionalización como una forma de repensar la universidad”. In: A. Bernasconi (ed.), La Educación Superior en Chile. Transformación, desarrollo y crisis. Santiago: Ediciones Universidad Católica de Chile, pp. 447-473.

MAURIN, Jost. (2005), "Flüchtlinge als politisches Instrument. Chilenische Emigranten in der DDR 1973-1989". Totalitarismus und Demokratie, n. 2, pp. 345-374.

MELLAFE, Rolando; GONZÁLEZ; María Teresa. (2007), El Instituto Pedagógico de la Universidad de Chile (1889-1981): su aporte a la educación, cultura e identidad nacional. Santiago de Chile: Universidad de Chile-Lom.

MÖBUS, Karlheinz. (2005a), “Die DDR und Chile Entdecken sich”. In: G. Schramm (ed.), Flucht vor der Junta. Die DDR und der 11. September. Berlin: Edition Ost, pp. 33-45.

. (2005b), "Chilenische Emigranten in der DDR". In: G. Schramm (ed.), Flucht vor der Junta. Die DDR und der 11. September. Berlin: Edition Ost, pp. 157-165.

MÜLLER-PLANTENBERG, Urs. (2002a), “Die chilenische Linke im deutschen Exil”. In: U. Müller-Plantenberg (ed.), Nachschläge. Ausgewählte politisch-soziologische Arbeiten 19972001. Münster, Lit Verlag, pp. 84-91.

. (2002b), "Aus der Frühzeit der Chile-Nachrichten". In: U. Müller-Plantenberg (ed.), Nachschläge. Ausgewählte politisch-soziologische Arbeiten 1997-2001. Münster: Lit Verlag, pp. 92-95.

OCDE. (2009), La Educación Superior en Chile. Revisión de las Políticas Nacionales de Educación. Santiago: Ministerio de Educación.

PATEL, Sujata. (2015), "The global transition an the challenge to social sciences". In: B. M. Bringel; J. M. Domingues (eds.): Global modernity and social contestation. Thousand Oaks, Ca.: Sage (Sage studies in international sociology), pp. 36-50. 


\section{Enrique Fernández Darraz}

PEDEMONTE, Rafael. (2010), "La "diplomacia cultural” soviética en Chile (1964-1973)". Bicentenario. Revista de Historia de Chile y América, v. 9, n. 1, pp. 57-100.

PORTALES, Carlos. (1989), Los factores externos y el régimen autoritario. Evolución e impacto de las relaciones internacionales de Chile en el proceso de transición a la democracia. (Documento de Trabajo, 419) Santiago, FLACSO.

POUTRUS, Patrice. (2015), "Zuflucht im Nachkriegsdeutschland. Politik und Praxis der Flüchtlingsaufnahme in Bundesrepublik und DDR von den späten 1940er Jahren bis zur Grundgesetzänderung im vereinten Deutschland von 1993". In: J. Oltmer (ed.), Handbuch Staat und Migration in Deutschland seit dem 17. Jahrhundert. Oldenburg: De Gruyter, pp. 853-893.

QUEZADA, Gonzalo. (1983), “Política exterior chilena 1958 - 1973: un análisis comparativo de flujos". Revista de Ciencia Política, v. 5 n. 1, pp. 48-76.

REBMANN, Linda. (2011), “La política alemana de cooperación para el desarrollo en la época de la Doctrina Hallstein y su incidencia en las relaciones con Brasil". Ciclos, v. 19, n. 37-38, pp. $137-160$.

RIVAS, Bernabé. (2009), "Polymere mit der Fähigkeit zur Abtrennung umweltverschmutzender Metalle”. In: A. von Humboldt Stiftung (ed.), Deutsch-Chilenische Forschungszusammenarbeit: Tradition und Zukunft. Bonn, Alexander von Humboldt Stiftung (Diskussionspapiere, 14), pp. 45-49.

RODRIGUEZ-MEDINA, Leandro. (2014), Circulation of European Knowledge: The Niklas Luhmann in the Hispanic Americas. New York: Palgrave Pivot.

ROJAS, Alvaro. (2009), "Die chilenisch-deutsche Wissenschaftskooperation: eine tragfähige Nasis für ein viel versprechender Zukunft". In: A. von Humboldt Stiftung (ed.), Deutsch-Chilenische Forschungszusammenarbeit: Tradition und Zukunft. Bonn, Alexander von Humboldt Stiftung (Diskussionspapiere, 14), pp. 9-11.

SANHUEZA, Carlos. (2010), "Circulación de intelectuales alemanes en Chile y chilenos en Alemania (fines del siglo XIX y comienzos del siglo XX)". Historia: Questoes $\mathcal{E}$ Debates, n. 53 , pp. $67-84$.

. (2011), “El debate sobre el embrujamiento alemán y el papel de la ciencia alemana por fines del siglo XIX en Chile". In: B. Göbel; G. Chicote (eds.), Ideas viajeras y sus objetos: el intercambio científico entre Alemania y América Austral. Madrid- Frankfurt, Iberoamericana / Vervuert.

. (2012), “El objetivo del Instituto Pedagógico no es formar geógrafos: Hans Steffen y la transferencia de saber geográfico alemán a Chile. 1893-1907". Revista Historia (Santiago), v. 45, n. 1, pp. 171-197.

SCHIDLOWSKY, David. (2008), Neruda y su tiempo: 1950-1973. Santiago: RiL Editores.

SCHÖLLGEN, Gregor. (2013), Deutsche Außenpolitik. München: Beck, C H.

SCHORIES, Dirk. (2009), "Im Zeichen von Wirtschaftskrise und Umweltproblemen gewinnt wissenschaftliche Zusammenarbeit mit Chile an Bedeutung". In: A. von Humboldt Stiftung (ed.), Deutsch-Chilenische Forschungszusammenarbeit: Tradition und Zukunft. Bonn, Alexander von Humboldt Stiftung (Diskussionspapiere, 14), pp. 18-22. 
SCHOTT, Thomas. (1993), "World science: globalization of institutions and participation". Science, Technology, \& Human Values, v. 18, n. 2, pp. 196-208.

SCHÜTTE, Georg. (2008), "Wettlauf ums Wissen: Außenwissenschaftspolitik als Herausforderung moderner Wissensgesellschaften". In: G. Schütte (ed.), Wettlauf ums Wissen. Aussenwissenchaftspolitik im Zeitalter der Wissensrevolution. Berlin, Berlin: University Press, pp. 12-26.

SCHÜTTE, Georg. (2010), "Außenwissenschaftspolitik. Wissenschaft im globalen Wandel gestalten". In: D. Simon; A. Kniew; S. Hornbostel (eds.), Handbuch Wissenschaftspolitik. Wiesbaden: VS Verlag für Sozialwissenschaften/GWV Fachverlage GmbH, pp. 151-161.

THESING, Josef. (2014), “Die Konrad-Adenauer-Stiftung in Lateinamerika: 50 Jahre politische Zusammenarbeit". In: D. Nolte (ed.), Internationale Parteienverbände und parteinahe Stiftungen in Lateinamerika. Baden-Baden, Nomos (Studien zu Lateinamerika, 27), pp. 211-245.

VAN KLAVEREN, Alberto. (2011), “La política exterior de Chile durante los gobiernos de la Concertación (1990-2010)". Estudios internacionales, n. 169, pp. 155-172.

VILLARROEL, Tomás. (2007), Las relaciones entre la República Federal Alemana y Chile (1952-1964). La política exterior de Alemania Federal hacia Chile bajo la marca del bipolarismo. Tesis (Magíster en Historia). Universidad de Chile, Santiago.

VON HUMBOLDT STIFTUNG, Alexander. (2009), "Deutsch-Chilenische Forschungszusammenarbeit: Tradition und Zukunft". Edited by Alexander von Humboldt Stiftung. Alexander von Humboldt Stiftung. Bonn (Diskussionspapiere, 14).

WALSH, Catherine. (2001), “¿Qué es conocimiento? Reflexiones sobre las políticas del conocimiento, el campo académico y el movimiento indígena ecuatoriano". En: Revista del Centro Andino de Estudios Internacionales, n. 2, pp. 65-77.

WENTKER, Hermann. (2007), Außenpolitik in engen Grenzen. Die DDR imp internationalen System, 1949-1989. München: Oldenbourg.

WERZ, Nikolaus. (2011), “Hinter der Mauer - Lateinamerika in der DDR". In: D. Brunner, Mario Niemann (eds.), Die DDR - eine deutsche Geschichte. Wirkung und Wahrnehmung. Schöningh: Paderborn, pp. 445-464.

WINNACKER, Ernst-Ludwig. (2008), "Zur internationalen Sichtbarkeit des deutschen Forschungsystem". In: G. Schütte (ed.), Wettlauf ums Wissen. Aussenwissenchaftspolitik im Zeitalter der Wissensrevolution. Berlin: Berlin University Press, pp. 85-91.

WISSENSCHAFTSRAT. (2010), Empfelungen zur deutschen Wissenschaftspolitik im Europäischen Forschungsraum. Berlin: Wissenschaftsrat. 


\section{Enrique Fernández Darraz}

\section{Fuentes Primarias}

Anales de la Universidad de Chile 1964 (AUCH)

BArch: Institut für Marxismus-Leninismus beim ZK der SED. Bundesarchiv, DY/30/IV A 2/9.07. Institut für Marxismus-Leninismus beim ZK der SED.

BArch: Internationale Verbindungen. Bundesarchiv, DY/30/IV A 2/20 726. Institut für Marxismus Leninismus beim ZK der SED.

Memorias del Ministerio de Relaciones Exteriores de Chile 1949-2014 (MRREE)

Prensa

Berliner Zeitung

El Siglo

Neue Zeit

Neues Deutschland

Fuentes de Información

www.bmbf.de

www.cinv.uv.cl

www.conicyt.cl

www.daad.de

www.dfg.de

www.fraunhofer.cl

www.heidelberg-center.uni-hd.de

www.hochschulkompass.de

www.hrk.de

www.leychile.cl

www.mecesup.cl

www.santiago.diplo.de 


\author{
RESUMO \\ Da Guerra Fria à Sociedade Global. As relações científicas chileno-alemãs 1949-2015: \\ contexto internacional e dinâmicas do intercâmbio.
}

O artigo analisa as dinâmicas das relações científicas chileno-alemãs desde o final da Segunda Guerra Mundial até hoje, inserindo-as no marco da discussão sobre a circulação de conhecimento e nas relações científicas internacionais. $\mathrm{O}$ texto tenta preencher lacunas da literatura atual, realizando uma análise em una perspectiva de longo prazo, integrando a todos os atores e localizando-as em um marco de política internacional amplo. Isso significa, não somente considerar aos atores tradicionais (Chile e a República Federal da Alemanha), mas também outros menos tratados (a República Democrática Alemã e o exílio chileno). Para desenvolver a análise foi feita uma revisão de arquivo das Memórias do Ministério de Relações Exteriores chileno. Também foi consultada a imprensa de ambos os países e documentos do Arquivo Federal alemão. Aprecia-se que as relações científicas chileno-alemãs desde o final da Segunda Guerra têm transitado por três etapas, cujas dinâmicas têm estado condicionadas pelas circunstâncias políticas internas, pelo contexto internacional e pelo desenvolvimento de seus próprios sistemas de educação superior e ciência. Nesta evolução, as relações científicas têm passado da dependência académica bastante tradicional a outra, focalizada em áreas científicas específicas do "centro".

Palavras-chave: relações internacionais; Chile; Alemanha; dependência académica; relações científicas.

\title{
ABSTRACT \\ From the Cold War to the Global Society. Chilean-German scientific relations 1949-2015: international context and dynamics of exchange.
}

This article analyzes the dynamics of Chilean-German scientific relations from the end of World War II until today, placing them in the framework of the discussion on the circulation of knowledge as well as in international scientific relations. The text attempts to fill gaps in current literature by conducting a long-term perspective analysis, integrated with all actors and located within a broad international policy framework. This means not only considering traditional actors (Chile and the Federal Republic of Germany), but also less debated ones (such as the German Democratic Republic and the Chilean exile). To develop the analysis, an archive review of the memoirs of the Chilean Ministry of Foreign Affairs was conducted. The press from both countries and documents from the German Federal Archive were also consulted. Chilean-German scientific relations since the end of World War II are understood to be organized in three stages, the dynamics of which have 


\section{Enrique Fernández Darraz}

been conditioned by internal political circumstances, the international context, and the development of their own higher education and science systems. In this evolution, scientific relations have shifted from quite traditional academic dependence to another paradigm, focused on specific "core" scientific areas.

Keywords: international relations; Chile; Germany; academic dependency; scientific relations.

\section{RÉSUMÉ}

De la Guerre Froide à la Société Mondiale: Relations scientifiques germano-chiliennes 1949-2015contexte international et dynamique des échanges.

Cet article analyse la dynamique des relations scientifiques entre l'Allemagne et le Chili depuis la fin de la Seconde Guerre mondiale jusqu'à aujourd'hui, en les plaçant dans le cadre du débat sur la circulation du savoir et les relations scientifiques internationales. Le texte tente de combler les lacunes de la littérature actuelle en effectuant une analyse dans une perspective à long terme, en intégrant tous les acteurs et en les plaçant dans un vaste cadre de politique internationale. Cela implique non seulement de prendre en compte les acteurs traditionnels (Chili et République Fédérale d'Allemagne), mais également les moins traités (la République Démocratique Allemande et l'exil chilien). Pour mener à bien l'analyse, un examen des dossiers des mémoires du ministère des Affaires étrangères du Chili a été effectué. La presse des deux pays et des documents des archives fédérales allemandes ont également été consultés. Il est à noter que les relations scientifiques entre l'Allemagne et le Chili depuis la fin de la Seconde Guerre mondiale ont connu trois étapes, dont la dynamique a été conditionnée par les circonstances politiques internes, par le contexte international et par le développement de leurs propres systèmes d'enseignement supérieur et de science. Au cours de cette évolution, les relations scientifiques sont passées d'une dépendance académique plutôt traditionnelle à une autre, axée sur des domaines scientifiques spécifiques $\mathrm{du}$ «centre».

Mots-Clés: relations internationales; Chili; Allemagne; unité académique; relations scientifiques. 


\section{RESUMEN}

De la Guerra Fría a la Sociedad Global. Las Relaciones Científicas Chileno-Alemanas 1949-2015: Contexto Internacional y Dinámicas del Intercambio

El artículo analiza las dinámicas de las relaciones científicas chileno-alemanas desde el fin de la Segunda Guerra Mundial hasta hoy, situándolas en el marco de la discusión sobre la circulación de conocimiento y las relaciones científicas internacionales. El texto intenta llenar vacíos de la literatura actual, realizando un análisis en una perspectiva de largo plazo, integrando a todos los actores y situándolas en un marco de política internacional amplio. Esto significa, no sólo considerar a los actores tradicionales (Chile y la República Federal Alemana), sino también a otros menos tratados (la República Democrática Alemana y el exilio chileno). Para desarrollar el análisis se hizo una revisión de archivo de las Memorias del Ministerio de Relaciones Exteriores chileno. También se consultó prensa de ambos países y documentos del Archivo Federal alemán. Se aprecia que las relaciones científicas chileno-alemanas desde el fin de la Segunda Guerra han transitado por tres etapas, cuyas dinámicas han estado condicionadas por las circunstancias políticas internas, por el contexto internacional y por el desarrollo de sus propios sistemas de educación superior y ciencia. En esta evolución, las relaciones científicas han pasado de una dependencia académica bastante tradicional a otra, focalizada en áreas científicas específicas, de mayor interés del "centro".

Palabras claves: relaciones internacionales; Chile; Alemania; dependencia académica; relaciones científicas 